“ (C) 2018 IEEE. Personal use of this material is permitted. Permission from IEEE must be obtained for all other uses, in any current or future media, including

reprinting/republishing this material for advertising or promotional purposes, creating new collective works, for resale or redistribution to servers or lists, or reuse of any copyrighted component of this work in other works." 


\title{
Pattern Reconfigurable, Vertically Polarized, Low-Profile, Compact, Near-Field Resonant Parasitic Antenna
}

\author{
Ming-Chun Tang, Senior Member, IEEE, Yunlu Duan, Zhentian Wu, Xiaoming Chen, Student Member, \\ IEEE, Mei Li, Member, IEEE, and Richard W. Ziolkowski, Fellow, IEEE
}

\begin{abstract}
A vertically polarized, low-profile, compact, near-field resonant parasitic (NFRP) antenna with pattern-reconfigurability is demonstrated. The antenna has three dynamic end-fire states facilitated with only three p-i-n (PIN) diodes. The radiation pattern in each state covers more than $120^{\circ}$ in its azimuth plane and, hence, it achieves beam-scanning that covers the entire azimuth plane. The antenna height and transverse size are, respectively, only $0.048 \lambda_{0}$ and $0.1 \lambda_{0}^{2}$. Measured results, in good agreement with their simulated values, demonstrate that the antenna exhibits a $\sim 11 \%$ fractional impedance bandwidth and a $\sim 6.6 \mathrm{dBi}$ peak realized gain in all three of its pattern-reconfigurable states. Stable and high peak realized gain values are realized over its entire operational band surrounding 2.22 GHz.
\end{abstract}

Index Terms-Compact, end-fire radiation, near-field resonant parasitic antenna, pattern-reconfigurable, vertical polarization.

\section{INTRODUCTION}

VERTICALLY polarized antennas with patternreconfigurability have garnered increasing attention lately. They provide several advantages which include improving signal-to-noise ratios, decreasing multipath fading, improving system performance characteristics, and saving energy. Reconfigurability allows for adjusting the main beams toward desired directions while suppressing unwanted beams in other directions. Furthermore, vertically polarized fields suffer much smaller attenuation losses than horizontal polarized ones when an electromagnetic wave propagates along a lossy medium such as the earth or realistic ground planes. This aspect makes them

Manuscript received on Jul. 31, 2018; revised on Nov. 5, 2018; and accepted on Nov. 17, 2018...

This work was supported in part by the National Natural Science Foundation of China contract number 61471072, in part by the Funding of the Innovative Leading Talents in Science and Technology of Chongqing contract number CSTCCXLJRC201705, in part by the Funding of the leading research talent cultivation plan of Chongqing University contract number cqu2017hbrc1 A08, and in part by Funding of the Young Backbone Teachers in Colleges and Universities of Chongqing contract number 0307001104102, in part by the Fundamental Research Funds for the Central Universities contract number 2018CDQYTX0025, and in part by the Australian Research Council grant number DP160102219.

M. -C. Tang, Y. Duan, Z. Wu, X. Chen and M. Li are with the Key Laboratory of Dependable Service Computing in Cyber Physical Society Ministry of Education, College of Communication Engineering, Chongqing University, Chongqing 400044, China (E-mail: tangmingchun@cqu.edu.cn)

R. W. Ziolkowski is with the University of Technology Sydney, Global Big Data Technologies Centre, Ultimo NSW 2007, Australia (E-mail: Richard.Ziolkowski@uts.edu.au). suitable in applications in the complex electromagnetic environments. Consequently, they have been widely applied in a variety of wireless systems. Examples include fifth-generation (5G) wireless communications devices [1], on-body communication systems [2], avionics applications [3], radar platforms [4], and customer-premises equipment (CPE) applications [5].

Considerable efforts have been devoted recently to realize vertically polarized antennas with pattern-reconfigurability. The existing techniques in extensive use can be generally classified into three categories. The first includes the integration of switchable ON/OFF devices such as p-i-n (PIN) [1], [2], [6]-[9] and varactor [4], [10]-[14] diodes. The second encompasses the introduction of switch networks such as controllable reactance circuits [15]-[16], beamforming networks [5], and control circuits [17]-[19]. The third resorts to mechanically controlled devices, e.g., movable liquid metals such as mercury [20] and electric motors to rotate the radiators [21]. While effective, these reconfigurable design schemes [1]-[4], [6]-[19] all consist of one central driven element and multiple parasitic elements around it. When operating in a single state, these reconfigurable systems only have a certain number of their parasitic elements active while the rest of them are idle. However, even though they are inactive, the additional space they occupy inescapably increases the total size of the antenna. On the other hand, when all the driven and parasitic elements are active at the same time, such as they are in each state in [5], a compact system is obtained. Nonetheless, it required a rather complex beamforming network to enable the requisite phase shifts to dynamically reconfigure its radiation patterns.

In this paper, a low-profile, compact, near-field resonant parasitic (NFRP) antenna that radiates vertically polarized, pattern-reconfigurable end-fire fields is reported. In Section II, two types of vertically polarized fan-shaped top-loaded folded monopole antennas are demonstrated and compared. One configuration includes the driven folded monopole and a single fan-shaped top-loaded monopole that acts as a NFRP element. The other has two such NFRP elements. Electrically small sizes ( $k a<1$, where $a$ is the radius of the smallest sphere that completely encloses the antenna system at the lower bound of the operational frequency range, $f_{L}$, and $k=2 \pi / \lambda_{L}=2 \pi f_{L} / \mathrm{c}$ is the corresponding free space wavenumber) are accomplished by loading the fan-shaped top-hats with U-shaped slots. These two 
antennas are shown to have excellent end-fire performance. It is demonstrated that the 3-element design achieves higher peak realized gain values with smaller fluctuations, i.e., its realized gain values are more stable, over its entire operational frequency band than the 2-element one. A pattern-reconfigurable 3-element system that covers the entire $360^{\circ}$ azimuth plane is then introduced in Section III. The design incorporates three of the fan-shaped top-loaded folded monopoles in a compact configuration. Its reconfigurability relies on a feed structure with only 3 PIN diode switches. As described in Section IV, a prototype of the optimized design was fabricated and tested to verify its simulated performance characteristics. The measured results, in good agreement with their simulated values, demonstrate that this compact, low profile system realizes stable peak realized gain values over its entire operational fractional bandwidth (FBW) for each of its pattern-reconfigurable states. Finally, some conclusions are drawn in Section V.

All the metallic elements in the antenna designs reported in this paper were chosen to be copper with its known material parameters: $\varepsilon_{r}=1.0, \mu_{r}=0.999991$ and bulk conductivity $\sigma=$ $5.8 \times 10^{7} \mathrm{~S} / \mathrm{m}$. All the numerical simulations and their optimizations were performed using the frequency domain ANSYS/ANSOFT high frequency structure simulator (HFSS), version 13.0. The $\left|S_{11}\right|$ (reflection coefficient) values for all of the fabricated prototypes as functions of the source frequency were measured using an Agilent E5063A PNA VNA. Their far-field radiation performance characteristics were measured in an anechoic chamber at the China Academy of Information and Communications Technology, Chongqing, China.

\section{Performance Comparison Between Two Types Of ELECTRICALLY SMALL, NFRP ANTENNAS}

The pattern-reconfigurable system followed from two NFRP antenna designs. Combining a driven folded monopole structure with one and two NFRP elements, two vertically polarized low-profile, electrically small, end-fire systems were obtained. Their design features and performance characteristics are characterized and compared. The physical mechanisms that led to the final design choices and that facilitated the pattern-reconfigurable system are explained.

\section{A. Configurations}

The one and two NFRP element configurations are shown, respectively, in Figs. 1(a) and 1(b). The corresponding optimized design parameters are detailed, respectively, in Tables I(a) and I(b). Fig. 1 illustrates that both antenna systems consist of two substrate boards. These copper-cladded substrate boards are Taconic RF-35 with a relative dielectric constant: $\varepsilon_{r}$ $=3.5$, loss tangent: $\tan \delta=0.0018$, and copper cladding thickness: $0.017 \mathrm{~mm}$. Both boards are circular with a $21 \mathrm{~mm}$ radius and have the same thickness, $0.76 \mathrm{~mm}$. The various conducting surfaces are etched on the top surfaces. The copper cladding on the lower surface of the lower substrate remains. It is directly connected to a copper ground plane disc whose radius is $30 \mathrm{~cm}$ and whose thickness is $1 \mathrm{~mm}$. A KTG 141-50 type, semi-rigid, $50 \Omega$ coaxial cable was selected to feed each of the two antennas. The radii of its inner and outer conductors are 0.47 and $1.79 \mathrm{~mm}$, respectively.
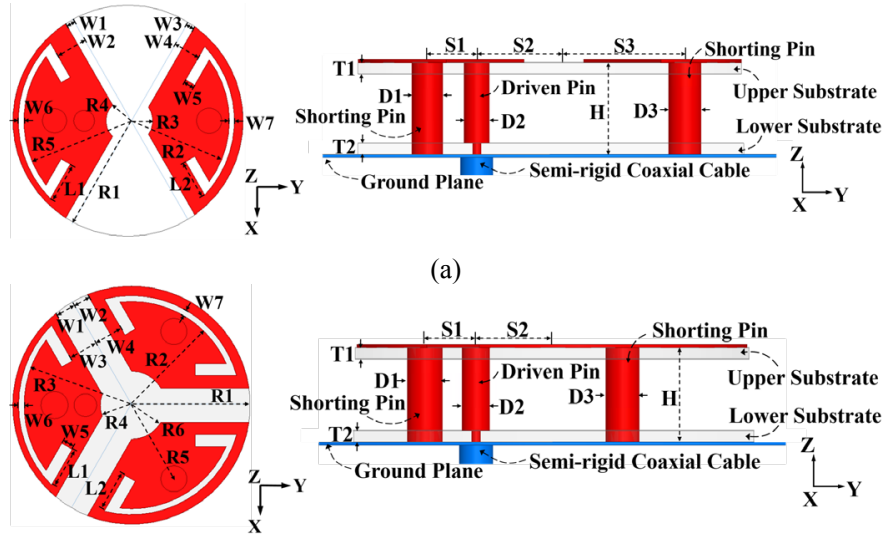

(a)

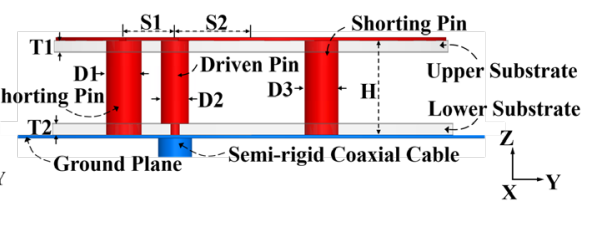

(b)

Fig. 1 Two vertically polarized NFRP antennas. Both have a driven fan-shaped top-loaded folded monopole element. They consist of (a) one and (b) two fan-shaped top-loaded monopole NFRP elements. Each of the fan-shaped components includes a U-shaped slot.

As shown in Fig. 1 (a), the 2-element NFRP antenna consists of two modified monopole elements. The first is the driven folded monopole. It is augmented with a fan-shaped top-hat that has a U-shaped slot etched into it. The top-hat is shorted to the ground plane by a cylindrical copper column (marked as the shorting pin) whose height is $H=6.5 \mathrm{~mm}$ and diameter is $D 1=$ $4.4 \mathrm{~mm}$. Thus, it penetrates through the bottom substrate. A second column (marked as driven pin) is connected directly to the top-hat. Its length and diameter are, respectively, $5.74 \mathrm{~mm}$ and $3.8 \mathrm{~mm}$. Both of these columns penetrate the substrate and are connected directly to the fan-shaped top-hat. The driven pin is connected directly to the center conductor of the coax and, hence, protrudes above the ground plane by $0.76 \mathrm{~mm}$. The NFRP element is a fan-shaped top-hat loaded monopole element. It is directly connected to the upper surface of the top-hat and to the ground plane by the shorting pin. Its height is also $6.5 \mathrm{~mm}$ and has a $D 3=4.6 \mathrm{~mm}$ diameter. When this two element antenna is operating, the juxtaposition of the driven and NFRP monopole elements produces end-fire radiation as anticipated in [22].

In contrast, the 3 -element NFRP antenna shown in Fig. 1 (b) consists of a similar driven element and two similar NFRP elements. All of the columns, the fan-shaped top-hat components and their U-shaped slots have been modified to fit them all into the same area. When the antenna is operating, it also produces excellent end-fire radiation. Because it has preferred performance characteristics, this 3-element NFRP antenna was fabricated and tested.

\section{B. Performance Characteristics}

The two NFRP antennas were optimized through simulations. Comparisons of the $\left|\mathrm{S}_{11}\right|$ values between the two antennas are 
shown in Fig. 2. The -10-dB bandwidth of the 2- (3-) element antenna is 125 (116) MHz from 2.143 (2.149) to 2.268 (2.265) $\mathrm{GHz}$ with the $\mathrm{FBW}=5.7 \%(5.3 \%)$. The realized gain and radiation efficiency (RE) values over the -10 -dB bandwidth region are depicted in Fig. 3. The maximum and minimum realized gain values of the 2- (3- ) element antenna are 7.37 (7.73) $\mathrm{dBi}$ and 4.65 (5.94) $\mathrm{dBi}$, respectively. The difference between the maximum and minimum realized gain values of the 3-element antenna is $1.79 \mathrm{dBi}$, while it is $2.72 \mathrm{dBi}$ for the 2element one. The simulated RE values for the two antennas are both higher than $90 \%$. Consequently, the fluctuations in the realized gain values within the operational frequency range of the 3-element antenna are smaller than those for the 2-element one for the same electrical size and height and very similar impedance bandwidths.

TABLE I THE Optimized Design PaRAMETERS OF THE TWO VerTiCALly POLARIZED NFRP ANTENNAS (IN MILLIMETERS)

(a) 2-Element Antenna

\begin{tabular}{|c|c|c|c|}
\hline$W 1=1$ & $W 2=5.2$ & $W 3=2$ & $W 4=5.4$ \\
\hline$W 5=1.9$ & $W 6=1$ & $W 7=0.9$ & $R 1=21$ \\
\hline$R 2=18.5$ & $R 3=4.5$ & $R 4=4$ & $R 5=19$ \\
\hline$D 1=4.4$ & $D 2=3.8$ & $D 3=4.6$ & $L 1=7.21$ \\
\hline$L 2=6.93$ & $T 1=0.76$ & $T 2=0.76$ & $S 1=8$ \\
\hline$S 2=5.4$ & $S 3=14.8$ & $H=6.5$ & Null \\
\hline
\end{tabular}

(b) 3-Element Antenna

\begin{tabular}{|c|c|c|c|}
\hline$W 1=3.5$ & $W 2=3$ & $W 3=5.2$ & $W 4=5.2$ \\
\hline$W 5=2.1$ & $W 6=0.95$ & $W 7=1$ & $R 1=21$ \\
\hline$R 2=18.3$ & $R 3=19$ & $R 4=5.5$ & $R 5=15$ \\
\hline$R 6=6$ & $D 1=4.8$ & $D 2=4$ & $D 3=4.6$ \\
\hline$L 1=7.26$ & $L 2=7.29$ & $T 1=0.76$ & $T 2=0.76$ \\
\hline$S 1=8.15$ & $S 2=5.45$ & $H=6.5$ & Null \\
\hline
\end{tabular}

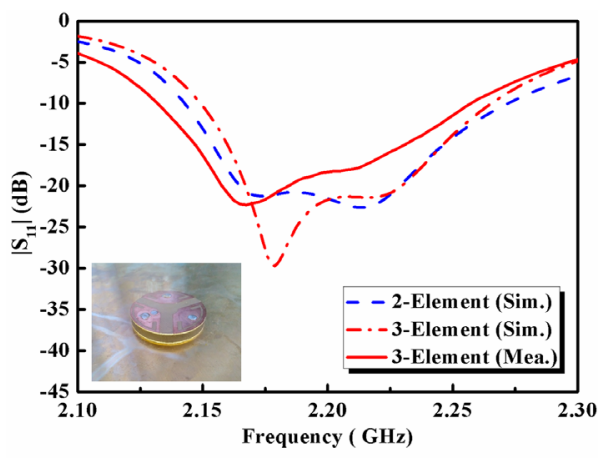

Fig. 2 Comparisons of the simulated and measured $\left|S_{11}\right|$ (reflection coefficient) values of the 2-element and 3-element vertically polarized NFRP antennas as functions of the source frequency.

The normalized realized gain patterns of the 2- and 3-element antennas are shown in Fig. 4 at their lower and higher resonance frequencies within their bandwidths. It is noted that the offset driven element and the presence of the large ground plane $(2.2$ $\lambda_{0}$ radius) causes the main beam to be tilted down $\sim 50^{\circ}$ from the normal to the ground in the $E$-plane ( $y z$-plane). It is clear from the $H$-plane ( $x y$-plane) that both antennas exhibit similar end-fire radiation characteristics over their entire operational frequency range. The half power beamwidths for both antennas is $\sim 148^{\circ}$ in $H$-plane, centered on the $-y$-axis. Because it has higher realized gain values with smaller fluctuations within its operational bandwidth, the 3-element NFRP antenna was fabricated, assembled, and measured. This prototype's measured $\left|S_{11}\right|$ and realized gain values, and its normalized realized gain patterns are also presented in Figs. 2-4 for comparison. The measured $-10-\mathrm{dB}$ bandwidth of the 3-element antenna is $122 \mathrm{MHz}$ from 2.133 to $2.255 \mathrm{GHz}$ with the $\mathrm{FBW}=$ $5.6 \%$. The maximum and minimum realized gain values are 7.9 and $6.1 \mathrm{dBi}$, respectively. Thus, the difference between its maximum and minimum realized gain values is only $1.8 \mathrm{~dB}$. The measured results are in reasonable agreement with their simulated values.

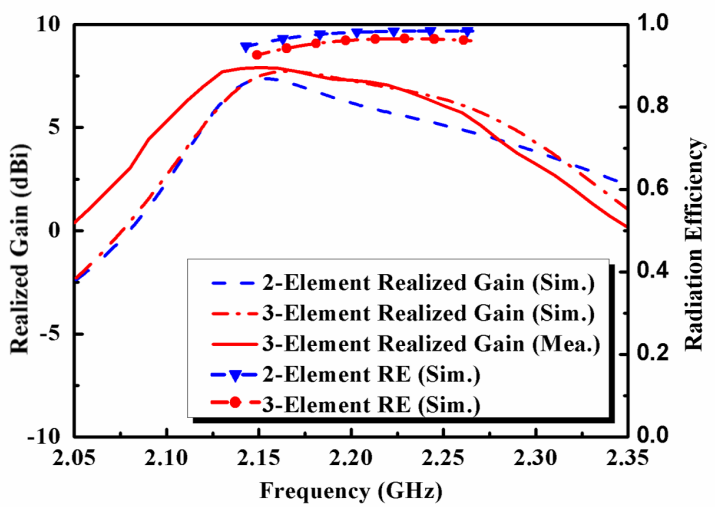

Fig. 3 Comparisons of the realized gain and radiation efficiency values of the 2-element and 3-element vertically polarized NFRP antennas as functions of the source frequency.

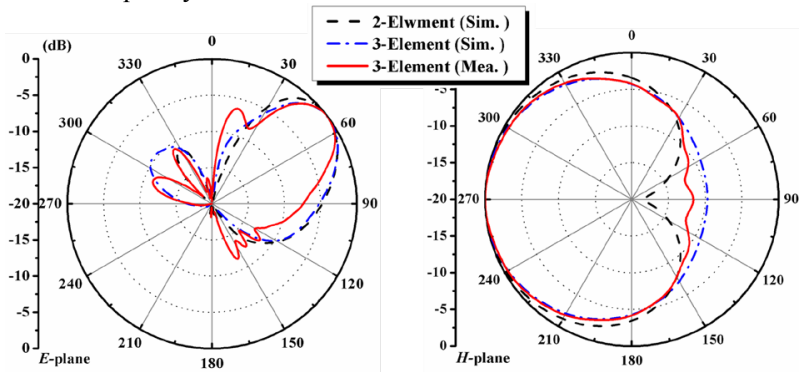

(a) Lower resonance frequency

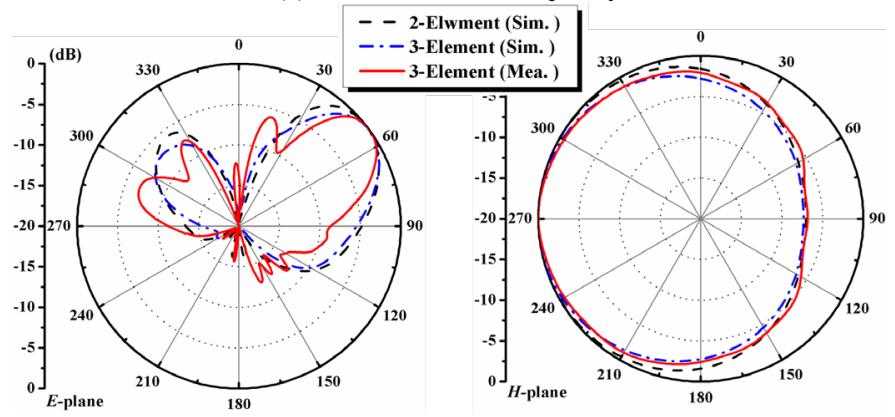

(b) Higher resonance frequency

Fig. 4 Comparisons of the simulated and measured normalized realized gain patterns $\left(\phi=270^{\circ}\right.$ in the E-plane, and $\theta=50^{\circ}$ in the H-plane) of the 2-element and 3-element NFRP antennas at two resonance frequencies. (a) Lower simulated (measured) resonance frequency: 2.17 (2.18) GHz. (b) Higher simulated (measured) resonance frequency: $2.21(2.22) \mathrm{GHz}$.

C. Physical Mechanisms

In order to illustrate the operational mechanisms of both antennas, the amplitude distributions of the surface currents on 
their upper conducting surfaces at two resonance frequencies are compared in Fig. 5. On the one hand, it is clear that the currents on the NFRP components in the 2-element antenna are much less than those on the 3-element one, especially in the higher operational frequency range. This feature addresses the smaller 2-element realized gain values. On the other hand, the end-fire performance arises from a quasi-Yagi behavior between the driven and NFRP elements [23, 24]. For a two-element Yagi, it is well-known [25] that end-fire radiation will occur if the driven and parasitic elements are separated by a distance of $\lambda / 4$ and have a $\pi / 2$ phase difference between them.



(I)

(II)

(a)

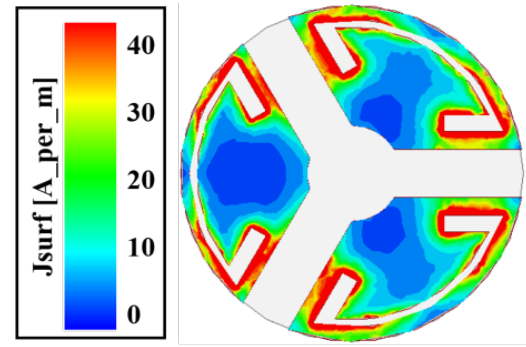

(I)



(II) (b)

Fig. 5 Amplitude distributions of the surface currents on the top-hat components of the (a) 2-element and (b) 3-element NFRP antennas at two frequencies. (I) Lower resonance (GHz), a: $2.17, b: 2.18$. (II) Higher resonance (GHz), a: 2.21 and b: 2.22 .

The introduction of the fan-shaped top-hats rather than the common circular ones [2], [7], facilitates the electrically small size of the systems. The introduction of the U-shaped slots localizes the surface currents to their edges as seen in Fig. 5 [26]. The combination of these features produces the requisite phase difference between the driven and NFRP elements to yield the end-fire behavior. The optimized designs make this particularly true at the lower resonant frequency of the 2- (3-) element antenna, 2.17 (2.18) GHz. However, this also means that the phase difference at the corresponding higher resonance frequency $2.21(2.22) \mathrm{GHz}$ is not quite right to maximize the end-fire behavior and the peak of the realized gain in Fig. 3 witnesses a decline there. It is also clearly observed in Fig. 5 that the magnitudes of the surface currents on the 3-element antenna components decrease much less than on the 2-element one at the higher frequency. This behavior is responsible for the more stable performance, i.e., the smaller realized gain fluctuations, of the 3-element design at the higher source frequencies.



(a)

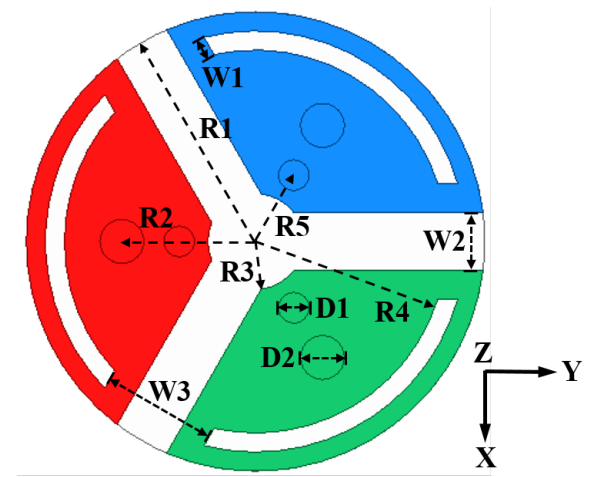

(b)



(c)

Fig. 6 The pattern-reconfigurable, vertically polarized NFRP antenna configuration. (a) 3-D isometric view. Top view of (b) the conducting fan-shaped components and (c) the feed structure now present on the upper surface of the lower substrate.

\section{PATtern-REConfigurable Vertically POLARIZED NFRP ANTENNA DESIGN}

Given it performance characteristics, the three-element NFPR antenna was selected for redesign to achieve a pattern-reconfigurable version. This design is illustrated in Fig. 6 . The corresponding optimized design parameters are given in Table II. 
TABLE II

THE OPTIMIZED DESIGN PARAMETERS OF THE PATTERN-RECONFIGURABLE VERTICALLY POLARIZED NFRP ANTENNA (IN MILLIMETERS)

\begin{tabular}{|c|c|c|c|}
\hline$R 1=24$ & $R 2=13.8$ & $R 3=5$ & $R 4=20$ \\
\hline$R 5=8.5$ & $W 1=2.2$ & $W 2=6$ & $W 3=12$ \\
\hline$W 4=1.5$ & $W 5=3.5$ & $W 6=2.05$ & $W 7=2.05$ \\
\hline$D 1=3.2$ & $D 2=4.6$ & $D 3=4.2$ & $G 1=0.4$ \\
\hline$G 2=0.5$ & \multicolumn{3}{|c|}{ Null } \\
\hline
\end{tabular}

The antenna is constructed with the same $0.76 \mathrm{~mm}$ thick Taconic RF-35 substrates and the same $1.0 \mathrm{~mm}$ thick ground plane. The radiating elements are three fan-shaped top-hat loaded folded monopoles. They are displayed in red, blue, and green in Fig. 6 for ease of description. Their conducting components were realized with the same printed circuit board (PCB) technology as the 3-element prototype was. The reconfigurable feed structure is located on the top surface of the lower substrate.

Each folded monopole consists of a driven pin, a shorting pin, and a fan-shaped top-hat with a C-shaped slot etched in it. In contrast to the 3-element antenna, the driving pin of each of these monopole structures is directly connected to a component of the reconfigurable feed structure. The center of the feed structure is directly connected to the center conductor of the KTG 141-50 coaxial cable. The external conductor of coaxial cable is again connected to the ground plane. All three monopole structures are identical to achieve the same performance characteristics in their respective $120^{\circ}$ sectors. For each of these states, one monopole structure is driven and the other two act as NFRP elements. The C-shaped slots not only simplify the design, but they also facilitate the necessary current magnitudes and phase differences to recover the end-fire radiation feature in each state. Moreover, because of the increased length of the driven pin which decreases the operational frequency, their smaller total lengths help to increase it and, hence, to overlap with the frequency region in which this end-fire radiation performance occurs.

As is shown in Fig. 6 (c), the feed structure consists of three pathways on the top surface of the bottom disc. They are symmetrically emanating from the center of the disc and are designed to accommodate pattern-reconfigurability into the three sectoral states. The three feed lines are metal branches, each containing a PIN diode, a capacitor, and two inductors. All three diodes are M/A-COM MA4GP907 PIN diodes [27]. According to its datasheet, each of these PIN diodes serves as a $4.0 \Omega$ resistor in its $\mathrm{ON}$ state and as a $0.025 \mathrm{pF}$ capacitor in its OFF state [27]. These diodes are denoted here as PIN_1, PIN_2, and PIN_3, respectively. Their negative poles are all set towards the disc center as depicted in Fig. 6(c). All three pairs of inductors were Murata $100 \mathrm{nH}$ coil inductors. Three Murata $10 \mathrm{pF}$ capacitors were employed to avoid any occurrence of short-circuit currents between the dc bias network and the vector network analyzer (VNA). In order to ensure the PIN diode locations during fabrication of the prototype, four small rectangular slots were etched on both sides of each diode position as markers.
The ON/OFF switch operations of the PIN diodes result in one folded monopole structure acting as the driven element and the other two folded monopole structures acting as the NFRP reflector elements [22-24]. By controlling the ON/OFF states of the three PIN diodes, the main beam direction is dynamically switched. In particular, when PIN_1 is turned ON and PIN_2 and PIN_3 are turned OFF, the RF signal passes through the red driven pin to directly excite the red folded monopole structure. As a consequence, the end-fire radiation pattern points along the red folded monopole structure direction ( $-y$-axis). We denote this state as State-1. In a similar manner, when PIN_2 (PIN_3) is $\mathrm{ON}$ and the other two are $\mathrm{OFF}$, the end-fire radiation pattern points along the blue (green) folded monopole structure direction; this state is labeled as State-2 (State-3). These three states, together with their PIN diode states, are summarized in Table III. Clearly, this three diode configuration makes the reconfigurable structure simple. The collocation of all three elements, one active and two passive, facilitates the system's compactness.

TABLE III

PIN Diode States For THE THREE PATTERN STATES

\begin{tabular}{|c|c|c|c|}
\hline State & PIN_1 & PIN_2 & PIN_3 \\
\hline 1 & ON & OFF & OFF \\
\hline 2 & OFF & ON & OFF \\
\hline 3 & OFF & OFF & ON \\
\hline
\end{tabular}

\section{Simulated AND MEASURED PERFormancE CHARACTERISTICS}

The optimized pattern-reconfigurable vertically polarized NFRP antenna was fabricated, assembled, and measured. A photograph of its components before assembly is presented in Fig. 7 (a). As shown in Fig. 7 (b), three different pairs of dc bias lines were used to control the ON/OFF states of the three PIN diodes. In practice, three blue wires were connected via the inductors to the negative poles of the three PIN diodes and three yellow wires were connected via the inductors to their positive poles. Thus, each pattern state employs one yellow ("+" pole) and all three blue ("-" pole) dc lines. An ATTEN TPR3003T-3C regulated dc power supply was used to feed the yellow wires with $\sim 1.4 \mathrm{~V}$ voltage to turn on the PIN diodes. The antenna under test (AUT) in the chamber is shown in Fig. 7 (b) along with this dc source and the assembled antenna with its dc bias lines.

As is shown in Fig. 7(b), a large extended copper ground plate was employed in the measurements to accurately evaluate its vertical polarization performance [3], [4], [6], [7], [14], [19]. The measured results together with their simulated values in each pattern state are presented in Fig. 8. Note that due to the antenna's strict symmetry, the simulated $\left|S_{11}\right|$ performance for each of the three states is the same. The corresponding measured performances are very much the same. 


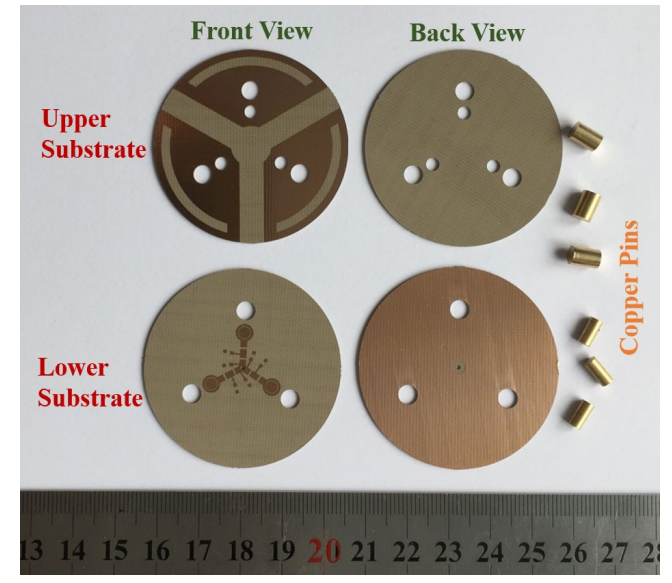

(a)

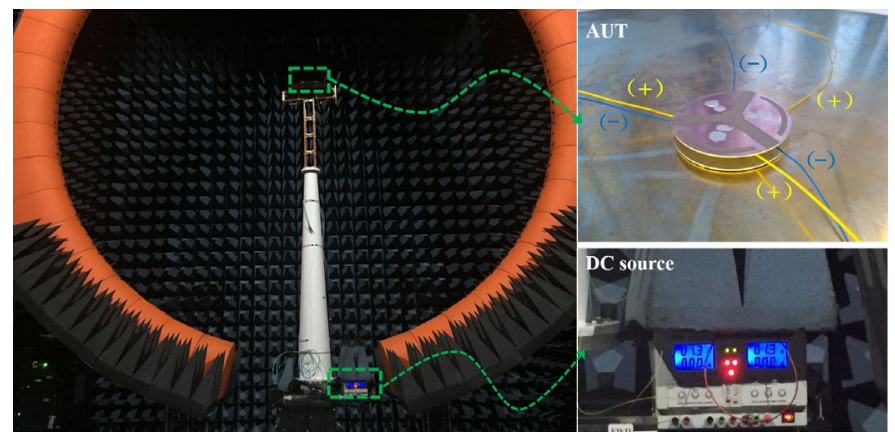

(b)

Fig. 7 Fabricated prototype of the pattern-reconfigurable vertically polarized antenna. (a) Antenna elements before assembly and (b) prototype antennas as the AUT in the chamber.

The simulated -10-dB impedance bandwidth in each state is 2.1 to $2.342 \mathrm{GHz}$ (242 MHz), which yields $\mathrm{FBW}=10.9 \%$. The measured -10-dB impedance bandwidths for State-1, State-2 and State-3 are from 2.113 to $2.348 \mathrm{GHz}(235 \mathrm{MHz}, 10.5 \%$ ), from 2.09 to $2.335 \mathrm{GHz}$ (245 MHz, 11.1\%), from 2.065 to $2.323 \mathrm{GHz}(258 \mathrm{MHz}, 11.8 \%)$, respectively. Therefore, the corresponding measured overlapped operating bandwidth for all three states is $210 \mathrm{MHz}(\mathrm{FBW}=9.47 \%)$, from 2.113 to $2.323 \mathrm{GHz}$. On the whole, the measured results demonstrate good agreement with their simulated values.

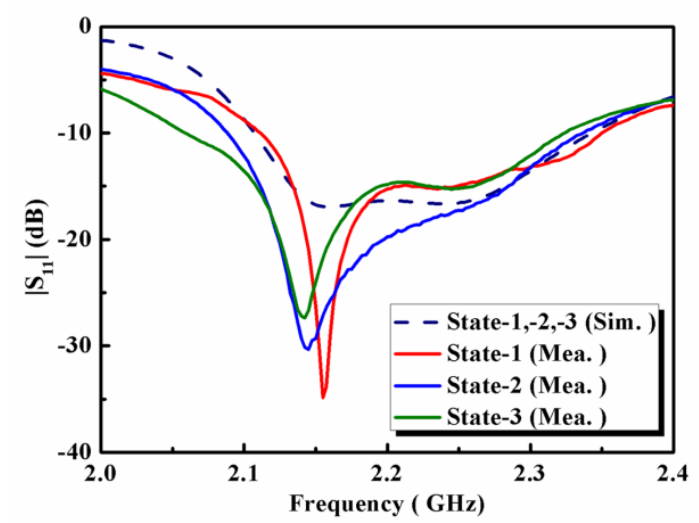

Fig.8 Simulated and measured $\left|\mathrm{S}_{11}\right|$ values of the pattern-reconfigurable vertically polarized antenna in its three pattern states.



(a) E-plane, $\phi=270^{\circ} ;$ H-plane, $\theta=54^{\circ}$

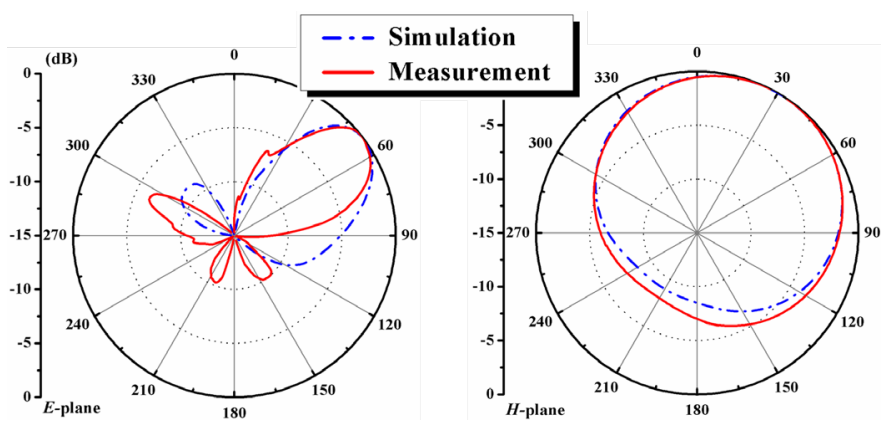

(b) E-plane, $\phi=30^{\circ} ;$ H-plane, $\theta=54^{\circ}$

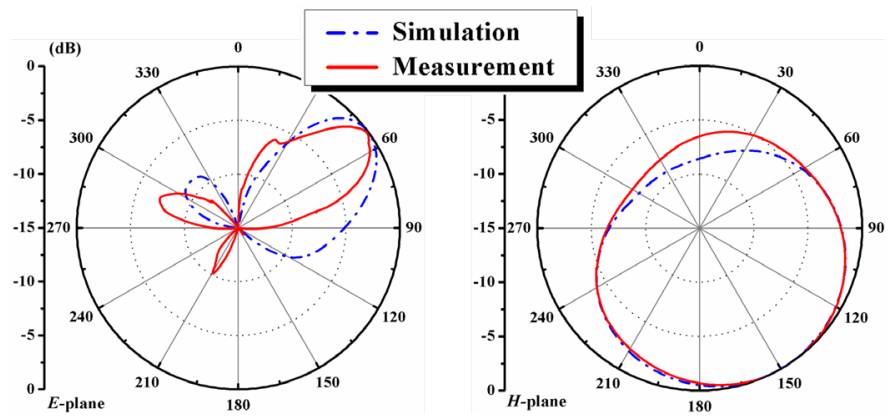

(c) E-plane, $\phi=150^{\circ} ; \mathrm{H}$-plane, $\theta=54^{\circ}$

Fig. 9 Simulated and measured normalized realized gain patterns when the antenna radiates each of its three states at the lower resonance frequency. (a) State-1. (b) State-2. (c) State-3

Fig. 9 presents the simulated and measured normalized realized gain patterns when the antenna operates in each of its pattern states at the selected frequency, $2.15 \mathrm{GHz}$, in its operational bandwidth. The main-beam direction is seen to point as expected from the three states listed in Table III. The simulated (measured) realized gain in State-1 is 7.13 (6.97) $\mathrm{dBi}$ and the simulated (measured) main-beam direction is pointed to $270^{\circ}\left(269^{\circ}\right)$ in the $H$-plane with a corresponding $160^{\circ}$ half-power beamwidth from $190^{\circ}$ to $350^{\circ}\left(159^{\circ}\right.$, from $187^{\circ}$ to $346^{\circ}$ ). Similarly, the simulated (measured) realized gain in State-2 is $7.13(6.36) \mathrm{dBi}$ and the simulated (measured) main-beam direction is pointed to $30^{\circ}\left(32^{\circ}\right)$ in the $H$-plane with a corresponding $160^{\circ}$ half-power beamwidth from $-50^{\circ}$ to $110^{\circ}$ $\left(161^{\circ}\right.$, from $-46^{\circ}$ to $\left.115^{\circ}\right)$. The simulated (measured) realized 
gain in State-3 is 7.13 (6.52) dBi and the simulated (measured) main-beam direction is pointed to $150^{\circ}\left(148^{\circ}\right)$ in the $H$-plane with a corresponding $160^{\circ}$ half-power beamwidth, from $70^{\circ}$ to $230^{\circ}\left(153^{\circ}\right.$, from $73^{\circ}$ to $\left.226^{\circ}\right)$.

TABLE IV

Measured and Simulated Performance Characteristics of the PatTERN-CONFIGURABLE VERTICALly POLARIZED NFRP ANTENNA

\begin{tabular}{|c|c|c|c|c|}
\hline \multicolumn{2}{|l|}{ State } & 1 & 2 & 3 \\
\hline \multirow{2}{*}{$\begin{array}{l}\text { Operational } \\
\text { frequency } \\
\text { range }(\mathrm{GHz})\end{array}$} & $\mathrm{S}$ & \multicolumn{3}{|c|}{$2.100-2.342$} \\
\hline & M & $2.113-2.348$ & $2.090-2.335$ & $2.065-2.323$ \\
\hline \multirow{2}{*}{$\begin{array}{l}\text { Bandwidth } \\
\text { (MHz) }\end{array}$} & $\mathrm{S}$ & \multicolumn{3}{|c|}{242} \\
\hline & M & 235 & 245 & 258 \\
\hline \multirow{2}{*}{$\begin{array}{c}\text { Fractional } \\
\text { bandwidth }(\%)\end{array}$} & $\mathrm{S}$ & \multicolumn{3}{|c|}{10.9} \\
\hline & $\mathrm{M}$ & 10.5 & 11.1 & 11.8 \\
\hline \multirow{2}{*}{$\begin{array}{l}\text { Peak realized gain } \\
(\mathrm{dBi})\end{array}$} & $\mathrm{S}$ & \multicolumn{3}{|c|}{7.13} \\
\hline & $\mathrm{M}$ & 6.97 & 6.36 & 6.52 \\
\hline \multirow{2}{*}{$\begin{array}{l}\text { Main-beam } \\
\text { direction }\end{array}$} & $\mathrm{S}$ & $270^{\circ}$ & $30^{\circ}$ & $150^{\circ}$ \\
\hline & $\mathrm{M}$ & $269^{\circ}$ & $32^{\circ}$ & $148^{\circ}$ \\
\hline \multirow{2}{*}{$\begin{array}{c}\text { 3-dB beam } \\
\text { coverage in } \\
\text { horizontal plane }\end{array}$} & $\mathrm{S}$ & $190^{\circ}-350^{\circ}$ & $-50^{\circ}-110^{\circ}$ & $70^{\circ}-230^{\circ}$ \\
\hline & M & $187^{\circ}-346^{\circ}$ & $-46^{\circ}-115^{\circ}$ & $73^{\circ}-226^{\circ}$ \\
\hline 3-dB beamwidth & $\mathrm{S}$ & \multicolumn{3}{|c|}{$160^{\circ}$} \\
\hline $\begin{array}{c}\text { in } \\
\text { horizontal plane }\end{array}$ & M & $159^{\circ}$ & $161^{\circ}$ & $153^{\circ}$ \\
\hline
\end{tabular}

The measured and simulated results of the pattern-reconfigurable vertically polarized NFRP antenna in all of its three pattern states are summarized in Table IV. On the whole, the average measured 3-dB beamwidth coverage in each dynamical state is $158^{\circ}$, much wider than $120^{\circ}$. Thus, as designed, the overlapping instantaneous $3-\mathrm{dB}$ beamwidths for all three states cover the entire azimuth plane. The measured results witness acceptable differences with their simulated ones. These small differences are attributed to several factors. First, because of the three PIN diodes are lumped elements and have manufacturing tolerances, their performance characteristics are not perfectly the same. Second, the long dc wires present in the measurement process were not considered in the simulations. Third, there were inevitable small dimension errors generated during the fabrication and assembly stages. Nonetheless, the measured and simulated values are in very reasonable agreement.

Table V compares the prototype's performance characteristics with those of previously reported vertically polarized antennas exhibiting entire azimuth plane coverage. Details include height, transverse electric size, gain, FBW, and number of states. Note that for a fair comparison, the total electrical size of each design corresponds to the center frequency of its $-10-\mathrm{dB}$ impedance bandwidth. These comparisons demonstrate that our antenna has several notable advantages. It is simple, utilizing the least number of PIN diodes. It is nearly the lowest profile. It provides a comparable operational bandwidth and peak gain values in nearly the smallest transverse size. It is noted that while the transverse size of the system reported in [19] is 12.5 times smaller, this was achieved by adding parasitic strips between the driven and reflector elements. Those additional elements made its height five times larger than our design. Moreover, it realized a narrower bandwidth and a worse level of impedance matching.

TABLE V

COMPARISON OF THE PATTERN-CONFIGURABLE VERTICALLY POLARIZED NFRP ANTENNA WITH RELATED ANTENNAS REPORTED IN THE LITERATURE

\begin{tabular}{|c|c|c|c|c|c|}
\hline Category & $\begin{array}{c}\text { Height } \\
\left(\boldsymbol{\lambda}_{\mathbf{0}}\right)\end{array}$ & $\begin{array}{c}\text { Transverse } \\
\text { size }\left(\boldsymbol{\lambda}_{\mathbf{0}}{ }^{2}\right)\end{array}$ & $\begin{array}{c}\text { Gain } \\
(\mathrm{dBi})\end{array}$ & $\begin{array}{c}\text { FBW } \\
(\mathbf{\%})\end{array}$ & $\begin{array}{c}\text { Number } \\
\text { of States }\end{array}$ \\
\hline Refs. & 0.38 & 0.12 & 4.9 & 8 & 6 \\
\hline$[1]$ & 0.04 & 0.28 & 6 & 5.7 & 4 \\
\hline$[3]$ & 0.25 & 1.31 & 12 & 16 & 12 \\
\hline$[4]$ & 0.09 & 0.14 & 6.7 & 19 & 4 \\
\hline$[5]$ & 0.25 & 0.69 & 8 & 19 & 6 \\
\hline$[6]$ & 0.048 & 0.52 & 7.74 & 10.8 & 3 \\
\hline$[7]$ & 0.13 & 0.22 & 6.5 & 20 & 12 \\
\hline$[8]$ & 0.5 & 2.25 & 10 & 14.5 & 6 \\
\hline$[9]$ & 0.09 & 0.11 & 4 & 10 & 6 \\
\hline$[10]$ & 0.1 & 0.11 & 4 & 4.1 & 6 \\
\hline$[11]$ & 0.08 & 0.20 & 7 & 8.2 & 6 \\
\hline$[13]$ & 0.063 & 0.22 & 1.5 & 5.7 & 4 \\
\hline$[14]$ & 0.18 & 0.14 & 5.1 & 17 & 6 \\
\hline$[18]$ & 0.24 & 0.008 & 7 & $<1$ & 8 \\
\hline$[19]$ & 0.048 & 0.10 & 6.6 & 11 & 3 \\
\hline This work & & & & \\
\hline
\end{tabular}

\section{CONCLUSION}

A low-profile, compact, end-fire, vertically polarized NFRP antenna with pattern-reconfigurability was presented. The antenna consists of three identical top-loaded folded monopoles and a reconfigurable feed structure to accommodate three overlapping end-fire pattern states. Their fan-shaped top-hats were augmented with $\mathrm{C}$-shaped slots to achieve its compact configuration, nearly complete impedance matching to its $50 \Omega$ source, and its end-fire radiation states. Only three PIN diodes were required; they were integrated into the feed structure to empower it pattern-reconfigurability. With one element on and the other two acting as NFRP elements, $\mathrm{a} \sim 158^{\circ}$ half-power beamwidth is achieved in the azimuthal plane with this 3 -element system in each of its three states, i.e., the radiation pattern in each of its reconfigurable states covers more than $120^{\circ}$ in the azimuth plane and, consequently, the system easily achieves a $360^{\circ}$ beam-scanning ability. A prototype of the optimized design was fabricated and measured. The simulated and experimental results are in good agreement. The antenna exhibits a $\sim 11 \%$ impedance bandwidth and a $\sim 6.6 \mathrm{dBi}$ peak realized gain in all of its pattern-reconfigurable states. Its height and transverse size were, respectively, only $0.048 \lambda_{0}$ and $0.1 \lambda_{0}{ }^{2}$. The prototype pattern-reconfigurable antenna has several potential applications in space-limited base stations and in compact intelligent transportation systems.

\section{REFERENCES}

[1] M. A. Hossain, I. Bahceci, and B. A. Cetiner, "Parasitic layer based radiation pattern reconfigurable antenna for $5 \mathrm{G}$ communications," IEEE Trans. Antennas Propag., vol. 65, no. 12, pp. 6444-6452, Dec. 2017.

[2] M. R. Kamarudin, Y. I. Nechayev, and P. S. Hall, "Onbody diversity and angle-of-arrival measurement using a pattern switching antenna," IEEE Trans. Antennas Propag., vol. 57, no. 4, pp. 964-971, Apr. 2009.

[3] L. Akhoobdzadeh-Asl, and J. J. Laurin, "Dual-mode L-band switched 
parasitic element antenna for avionics applications," in Proc. 2012 IEEE International Symposium on Antennas and Propagation, 8-14 July, 2012, Chicago, IL, pp. 1-2.

[4] H. Scott, and V. F. Fusco, " $360^{\circ}$ electronically controlled beam scan array," IEEE Trans. Antennas Propag., vol. 52, no. 1, pp. 333-335, Jan. 2004.

[5] W. Wan, G. Wen. and S. Gao, "Optimum design of low-cost dual-mode beam-steerable arrays for customer-premises equipment applications," IEEE Access, vol. 6, pp. 16092-16098, 2018.

[6] K. K. Katare, A. Biswas, and K. P. Esselle, "Directive array based pattern reconfigurable," in Proc. 2017 11th European Conference on Antenna and Propagation(EUCAP), 19-24 Mar., 2017, Paris, France, pp. 2029-2032.

[7] J. Q. Shi, Z. X. Hu, Z. X. Shen, and W. Wu, "A pattern reconfigurable low-profile Yagi monopole antenna with $360^{\circ}$ beam-scanning ability," in Proc. 2016 Asia-Pacific Microwave Conference(APMC), 5-9 Dec. 2016, New Delhi, India, pp. 1-3.

[8] H. T. Liu, S. Gao, and T. H. Loh, "Compact dual-band antenna with electronic beam-steering and beamforming capability," IEEE Antennas Wireless Propag. Lett., vol. 10, pp. 1349-1352, 2011.

[9] Y. Yang, and X. Zhu "A wideband reconfigurable antenna with $360^{\circ}$ beam-steering for 802.11 ac WLAN applications," IEEE Trans. Antennas Propag., vol. 66, no. 2, pp. 600-608, 2018.

[10] H. T. Liu, S. Gao, and T. H. Loh, "Electrically small and low cost smart antenna for wireless communication," IEEE Trans. Antennas Propag., vol. 60, no. 3, pp. 1540-1549, Mar. 2012.

[11] H. T. Liu, S. Gao, and T. H. Loh, "Compact-size electronically steerable parasitic array radiator antenna," in Proc. 2009 Loughborough Antennas \& Propagation Conference (LAPC), 16-17 Nov. 2009, Loughborough, UK, pp. 265-268.

[12] H. Kawakami, and T. Ohira, "Electrically steerable passive array radiator (ESPAR) antennas," IEEE Antennas Propag. Mag., vol. 47, no. 2, pp. 43-50, 2005.

[13] S. Kausar, H. U. Rahman, T. Hassan, and A. Kausar, "Miniaturization of ESPAR antenna using folded monopoles and conical central element," in Proc. 2015 International Conference on Radar, Antenna, Microwave, Electronics and Telecommunications (ICRAMET), 5-7 Oct. 2015, Bandung, Indonesia, pp. 87-91.

[14] L. Akhoobdzadeh-Asl, J. J. Laurin, and A. Mirkamali, "A novel low-profile monopole antenna with beam switching capabilities," IEEE Trans. Antennas Propag., vol. 62, no. 3, pp.1212-1220, Mar. 2014.

[15] R. Schlub, J. Lu, and T. Ohira, "Seven-element ground skirt monopole ESPAR antenna design from a genetic algorithm and the finite element method," IEEE Trans. Antennas Propag., vol. 51, no. 11, pp.3033-3039, Nov. 2003.

[16] T. D. Dimousios, C. I. Tsitouri, S. C. Panagiotou, and C. N. Capsalis, "Design and optimization of a multipurpose tri-band electronically steerable passive array radiator (ESPAR) antenna with steerable-beam-pattern for maximum directionality at the frequencies of 1.8, 1.9 and 2.4 GHz with the aid of genetic algorithms," in Proc. 2008 Loughborough Antennas and Propagation Conference, 17-18 Mar. 2008, Loughborough, UK, pp. 253-256.

[17] E. Palantei, and D. V. Thiel, "Symmetry problems in switched parasitic smart antennas," in Proc. 2007 IEEE Antennas and Propagation Society International Symposium (APS), 10-15 Jun. 2007, Honolulu, HI, pp. 3360-3363.

[18] J. Lu, D. Ireland, and R. Schlub, "Dielectric embedded ESPAR (DE-ESPAR) antenna array for wireless communications," IEEE Trans. Antennas Propag., vol. 53, no. 8, pp.2437-2443, Aug. 2005.

[19] Y. Juan, W. Che, W. Yang, and Z. N. Chen, "Compact pattern-reconfigurable monopole antenna using parasitic strips," IEEE Antennas Wireless Propag. Lett., vol. 16, pp. 557-560, 2017.

[20] D. Rodrigo, L. Jofre, and B. A. Cetiner, "Circular beam-steering reconfigurable antenna with liquid metal parasitics," IEEE Trans. Antennas Propag., vol. 60, no. 4, pp.1796-1802, Apr. 2012.

[21] H. L. Zhu, S. W. Cheung, and T. L. Yuk, "Mechanically pattern reconfigurable antenna using metasurface," IET Microwaves, Antennas \& Propagation, vol. 9, no. 12, pp 1331-1336, 2015.

[22] M.-C. Tang, and R. W. Ziolkowski, "Two-element Egyptian axe dipole arrays emphasizing their wideband and end-fire radiation performance," IET Microwaves, Antennas \& Propagation, vol. 9, no. 13, pp. 1363-1370, 2015.
[23] M.-C. Tang, R. W. Ziolkowski, S. Xiao, and M. Li, "A high-directivity, wideband, efficient, electrically small antenna system," IEEE Trans. Antennas Propag., vol. 62, no. 12, pp. 6541-6547, Dec. 2014.

[24] M.-C. Tang, T. Shi, and R. W. Ziolkowski, "Flexible efficient quasi-Yagi printed uniplanar antenna," IEEE Trans. Antennas Propag., vol. 63, no. 12, pp. 5343-5350, Dec. 2015

[25] C. A. Balanis, Antenna Theory: Analysis and Design, $4^{\text {th }}$ Ed., Hoboken, NJ, USA: John Wiley \& Sons, 2016, Sec. 6.2.

[26] R. W. Ziolkowski, and P. Jin, "Metamaterial-based dispersion engineering to achieve high fidelity output pulses from a log-periodic dipole array," IEEE Trans. Antennas Propag., vol. 56, no. 12, pp. 36193629, Dec. 2008

[27] MACOM. (2017). Products: MA4GP907. [Online]. Available: http://cdn.macom.com/datasheets/MA4GP907.pdf. 




Ming-Chun Tang (S'12-M'13-SM'16) received the B. S. degree in physics from the Neijiang Normal University, Neijiang, China, in 2005 and the Ph. D. degree in radio physics from the University of Electronic Science and Technology of China (UESTC), in 2013. From August 2011 to August 2012, he was also with the Department of Electrical and Computer Engineering, The University of Arizona, Tucson, AZ, USA, as a Visiting Scholar. He is currently a Professor in the College of Communication Engineering, Chongqing University, China. His research interests include electrically small antennas, RF circuits, metamaterial designs and their applications.

Prof. Tang was a recipient of the Best Student Paper Award in the 2010 International Symposium on Signals, Systems and Electronics (ISSSE2010) held in Nanjing, China. His Ph.D. student received Best Student Paper Award from the IEEE $7^{\text {th }}$ Asia-Pacific Conference on Antennas and Propagation (2018 IEEE APCAP) held in Auckland, New Zealand. He is the founding Chair of the IEEE AP-S / MTT-S Joint Chongqing Chapter. He serves on the Editorial Boards of several journals, including IEEE Access, IET Electronics Letters and IET Microwaves, Antennas \& Propagation. He has also served on the review boards of many journals, including the IEEE Transactions on Antennas and Propagation, IEEE Transactions on Microwave Theory and Techniques, IEEE Antennas and Wireless Propagation Letters, IEEE Antennas and Propagation Magazine, IEEE Microwave and Wireless Components Letters, and many international conferences as a General Chair, TPC Member, Session Organizer, and the Session Chair.

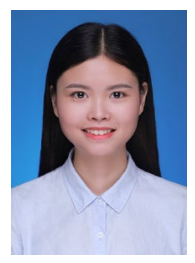

Yunlu Duan received the B.S. degree in electronics and information engineering from Chongqing University, Chongqing, China, in 2017. She is currently pursuing the M.S. degree in information and communication technology engineering with Chongqing University, Chongqing, China.

Her current research interests include electrically small antenna and vertical polarization antenna.

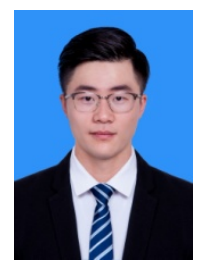

Zhentian Wu was born in Anhui, China, in 1994. He received the B.S. degree from the West Anhui University, Luan, China, in 2016. He is currently pursuing the M.S. degree in electronics and communication engineering in the College of Communication Engineering, Chongqing University, China.

He current research interests include electrically small antenna and directional antenna and their applications.

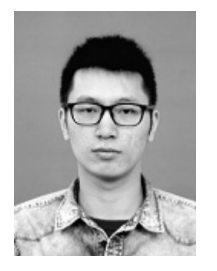

Xiaoming Chen (S'16) received the B.S. degree in electronics and information engineering from Chongqing University, Chongqing, China, in 2016. He is currently pursuing the $\mathrm{Ph}$. D. degree in information and communication technology engineering with Chongqing University, Chongqing, China.

His current research interests include intelligent algorithms in antenna design, circularly polarized antennas.

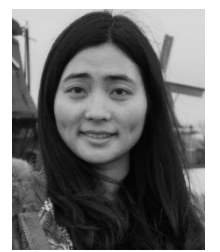

Mei Li (M'16) received the Ph.D. in radio physics from the University of Electronic Science and Technology of China, Chengdu, in 2016. From 2014 to 2016, she was with the Applied Electromagnetics Research Group, University of California at San Diego, San Diego, CA, USA, as a Visiting Graduate. She is currently with Chongqing University. Her current research interests include metasurfaces, antennas and arrays.

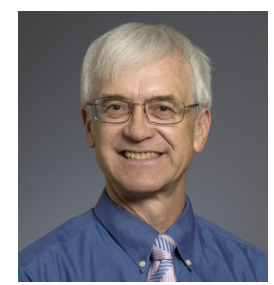

Richard W. Ziolkowski (M'87-SM'91-F'94) received the Sc. B. (magna cum laude) degree (Hons.) in physics from Brown University, Providence, RI, USA, in 1974; the M.S. and Ph.D. degrees in physics from the University of Illinois at Urbana-Champaign, Urbana, IL, USA, in 1975 and 1980, respectively; and an Honorary Doctorate degree, Doctor Technish Honoris Causa, from the Technical University of Denmark (DTU), Kongens

Lyngby, Denmark in 2012.

Prof. Ziolkowski is the recipient of the 2019 IEEE Electromagnetics Award (IEEE Field Award). He is a Fellow of the Optical Society of America (OSA, 2006) and the American Physical Society (APS, 2016). He served as the President of the IEEE Antennas and Propagation Society in 2005. He is also actively involved with the URSI, OSA and SPIE professional societies. He was the Australian DSTO Fulbright Distinguished Chair in Advanced Science and Technology from 2014-2015. He was a 2014 Thomas-Reuters Highly Cited Researcher.

He is a Distinguished Professor in the Global Big Data Technologies Centre in the Faculty of Engineering and Information Technologies (FEIT) at the University of Technology Sydney, Ultimo NSW, Australia. He became a Professor Emeritus at the University of Arizona in 2018, where he was a Litton Industries John M. Leonis Distinguished Professor in the Department of Electrical and Computer Engineering in the College of Engineering and also a Professor in the College of Optical Sciences. He was the Computational Electronics and Electromagnetics Thrust Area Leader with the Engineering Research Division of the Lawrence Livermore National Laboratory, Livermore, CA, USA, before joining The University of Arizona, Tucson, AZ, USA, in 1990. His current research interests include the application of new mathematical and numerical methods to linear and nonlinear problems dealing with the interaction of electromagnetic and acoustic waves with complex linear and nonlinear media, as well as metamaterials, metamaterial-inspired structures, nano-structures, and other classical and quantum applications-specific configurations. 